\title{
EFECTO DEL CAMBIO DE JAULAS EN LA PRODUCCIÓN DE RATAS SPRAgue DAWLEy
}

\author{
Edwin León-Mora*, Quim. ${ }_{1}$, Ledis Reyes-Moreno, M.Sc., Luis Mariano Pacheco-Salazar, Biol. ${ }_{1}$, Fátima \\ Caiza-Llumiquinga, M.Sc. ${ }_{1}$
}

${ }_{1}$ Laboratorio de Ensayos Biológicos (LEBi), Universidad de Costa Rica, San José, Costa Rica

Recibido: 27 de agosto del 2013 Aprobado: 20 de noviembre del 2013

* Autor de correspondencia: Edwin León Mora, Universidad de Costa Rica, Laboratorio de Ensayos Biológicos. Teléfono: (00506) 25114565. Correo electrónico: edwin.leon@ucr.ac.cr

Cómo citar este artículo: León-Mora E, Reyes-Moreno L, Pacheco-Salazar LM, Caiza-Llumiquinga F. Efecto del cambio de jaulas en la producción de ratas Sprague Dawley. Spei Domus. 2013; 9(19): 17-22.

Resumen. Las señales olfatorias que dejan las ratas madres para comunicarse con sus crías son críticas. Una mayor frecuencia de la limpieza de los recintos primarios eliminaría las señales olfatorias afectando el número de crías destetadas. Para medir la influencia de cambios en la producción de ratas de la cepa Sprague Dawley, se determinó cambiar de jaula a las hembras desde apareo hasta destete una vez a la semana ( 28 hembras), el cual se determinó como grupo tratamiento, y dos veces a la semana (28 hembras), el que se designó como grupo control, pues este es régimen habitual de cambio. Además se registraron los valores mínimos y máximos de temperatura y humedad del recinto secundario para comparar su influencia respecto al cambio de animales; esto comparado con el efecto del número de parto o el cambio de régimen ( 8 hembras de cada grupo). Se comparó la media de crías nacidas de las madres por medio de contraste de hipótesis y se analizó el efecto de la temperatura, la humedad y el efecto de un segundo parto por medio de regresión logística. Se obtuvo que al realizar el contraste de hipótesis no hay diferencia estadísticamente significativa en la cantidad de animales producidos al destete para uno o dos cambios. Y al comparar con los otros factores se obtiene que es significativo el número de parto de hembras en la cantidad de animales producidos en la regresión logística más que modificar el régimen de cambio de jaulas de los animales.

Palabras clave: frecuencia de cambio de jaulas, producción animal de laboratorio, recinto primario, Sprague Dawley.

\section{Effects of Cage Change on the Production of Sprague Dawley Rats}

Abstract. Olfactory signals that enable mother rats to communicate with their young are critical. More frequent cleaning of primary enclosures eliminates olfactory signals that affect the number of pups weaned. To measure the influence of changes on the production of rats of the Sprague Dawley strain, it was decided to change the cages of females once a week throughout the production cycle (from mating until weaning) (28 females) for the experimental group, and twice a week (28 females) for the control group because this is the usual system for changing them. The minimum and maximum temperature and humidity values of the secondary enclosure were also recorded to compare their influence on changes in the animals; this was then compared to the effect on the number of births or the change in the system ( 8 females in each group). The average number of pups born to mother rats was compared using hypothesis contrasting and the effects of temperature, humidity and a second delivery were analyzed using logistical regression. It was found that when performing hypothesis testing, there was no statistically significant difference in the number of animals produced at weaning due to one change or two changes. And when compared to the other factors, it was found that the number of females produced in the logistical regression was more significant than the modification in changing the animals' cages.

Keywords: cage change frequency, laboratory animal production, primary enclosure, Sprague Dawley.

\section{Efeito da mudança de jaulas na produção de ratos Sprague Dawley}

Resumo. Os sinais olfatórios que os ratos-matrizes deixam para se comunicarem com suas crias são críticas. Uma maior frequência da limpeza dos recintos primários eliminaria os sinais olfatórios, o que afeta o número de crias desmamadas. Para medir a influência de mudanças na produção de ratas da linhagem Sprague Dawley, determinou-se mudar de jaula as fêmeas desde o acasalamento até desmamentação uma vez por semana (28 fêmeas), o que se determinou como grupo tratamento, e duas vezes por semana (28 fêmeas), o que se designou como grupo controle, por ser este o regime habitual de mudança. Além disso, registraram-se os valores mínimos e máximos de temperatura e umidade do recinto secundário para comparar sua influência a respeito da mudança de animais; isso comparado com o efeito do número de parto ou a mudança de regime (8 fêmeas de cada grupo). Comparou-se a média de crias nascidas das mães por meio de contraste de hipótese e analisou-se o efeito da temperatura, da umidade e do efeito de um segundo parto por meio de regressão logística. Obteve-se que, ao realizar o contraste de hipótese, não há diferença significativa estatisticamente na quantidade de animais produzidos à desmamentação para uma mudança ou para duas mudanças. E, ao comparar com outros fatores, obtém-se que é significativo o número de parto de fêmeas na quantidade de animais produzidos na regressão logística mais que modificar o regime de mudança de jaulas dos animais.

Palavras-chave: frequência de mudança de jaulas, produção animal de laboratório, recinto primário, Sprague Dawley. 


\section{Introducción}

El cambio de jaulas o recinto primario es un factor primordial en el mantenimiento de animales de laboratorio, debido a que contribuye a disminuir el nivel de amoniaco presente [1]. El nivel aceptado de amoniaco para los humanos es de $25 \mathrm{ppm}$ por ocho horas de trabajo diario [2]. Sin embargo, no existen directrices oficiales para animales de laboratorio debido a la variación entre especie. Una unidad de animales puede estar potencialmente expuesta a amonio las 24 horas del día cada día. En roedores, así como en humanos, una exposición alta y prolongada a concentraciones de amoniaco puede causar daños oculares y respiratorios [3-6] y daños potenciales en la piel por contactos prolongados con la cama de las jaulas $[7,8]$.

Investigaciones previas demuestran que la limpieza regular de las jaulas es importante para la salud de las ratas, pero en el caso de las crías hay una alteración del nido y eliminación de señales olfativas que son importantes para el cuido materno de estas [9]. En frecuentes limpiezas de cajas el estrés crónico o acumulativo es mucho menos seguro. En ratones, dos estudios encontraron que la limpieza frecuente de las cajas incrementa la mortalidad de las crías [10, 11]. Se ha demostrado que el levantamiento periódico de las tapas de las jaulas provoca una fuerte tendencia hacia el éxito reproductivo más pobre [12].

Investigaciones previas en ratas Wistar y Sprague Dawley demostraron evidencias de que frecuentes limpiezas de las jaulas afecta el bienestar de los animales que están en experimentación [9]. En ratas en producción también se ha reportado que la frecuente limpieza tiene un efecto en el descenso en la cantidad de animales producidos a largo plazo. Se obtiene que, aunque las crías fueron destetadas con éxito, el estudio presenta un aumento en el canibalismo [13]. Se sugiere que en ratas preñadas se debe minimizar el régimen de limpieza de cajas, ruidos, olores no familiares hasta los primeros días después del nacimiento [14].

Esta investigación evalúa el efecto de la frecuencia de la limpieza de jaulas sobre la reproducción de ratas Sprague Dawley, animales utilizado por docentes, investigadores y estudiantes, producidos en el Bioterio del Laboratorio de Ensayos Biológicos de la Universidad de Costa Rica.

\section{Materiales y métodos}

\section{Animales}

Fueron utilizadas 56 ratas hembras de la cepa Sprague Dawley (Hsd: SD), nulíparas, provenientes del Bioterio del Laboratorio de Ensayos Biológicos (LEBi) de la Universidad de Costa Rica, con un peso corporal aproximado de 250 gramos, de tres a cuatro meses edad.

Los animales fueron mantenidos bajo las siguientes condiciones: iluminación, ciclos de 12 horas de luz y 12 de oscuridad; recambios de aire, de 10 a 15 cambios por hora; temperatura, 18 a $28{ }^{\circ} \mathrm{C}$ (máximo y mínimo reportados); humedad, 50-95\% (máximo y mínimo reportados); dimensiones de la jaula, $595 \mathrm{~mm}$ x $380 \mathrm{~mm}$ x $200 \mathrm{~mm}$.

Disposición: una hembra junto con sus crías por jaula. El agua consumida por los animales es acidificada a un $\mathrm{pH}$ 2,5 a 3,0 y se utiliza alimento para animales de laboratorio, Concentrados Aguilar \& Solís (Cargill S. A.), consumo ad libitum. Durante el desarrollo del estudio se realizan monitoreos microbiológicos ambientales, microbiológicos del agua y del alimento de consumo de los animales con una frecuencia trimestral.

Las ratas se alojan de manera individual en jaulas de policarbonato, marca Tecniplast, número de catálogo 1354G Eurostandard Type Iv (dimensiones de la jaula, 595 $\mathrm{mm}$ x $380 \mathrm{~mm}$ x $200 \mathrm{~mm}$ ) con una cama de viruta de madera y con un espesor aproximado del encamado de 3-5 $\mathrm{cm}$ mantenido constantemente en todo el estudio. La viruta se esteriliza antes de su uso en las jaulas de los animales. Las cajas de los animales se colocaron juntamente en un solo estante en todos los momentos de la investigación, colocando en el mismo las cajas control y tratamiento.

Los animales fueron manipulados siempre por el mismo técnico especializado en animales de laboratorio. El estudio fue aprobado por el Comité Institucional para el Cuido y Uso de Animales de la Universidad de Costa Rica (Cicua), número de aprobación 79-2008.

\section{Diseño experimental}

Se estableció para esta investigación un grupo control (28 animales), que se definió como el que mantiene un régimen de limpieza inalterado de los animales del que se acostumbra en el bioterio que es de dos veces 
semanales, realizando el cambio de cajas de los animales para las crías de menos de cinco días, tomándolas todas juntas con las dos manos y las hembras del lomo. Al grupo tratamiento se le establece un sólo cambio de cama semanal (28 animales). Se mantuvo la misma técnica de traspaso de animales que en el grupo control.

Para ambos grupos se mantuvo el correspondiente régimen de limpieza durante las etapas de apareo (una semana), gestación (tres semanas) y lactancia (tres semanas) del primer parto, y esto se repitió para 16 hembras ( 8 control y 8 tratamiento) escogidas al azar para un segundo parto de las mismas.

\section{Temperatura y humedad}

La temperatura fue medida con termohigrómetros (Cole Parmer, modelo: 03313-86) dentro del recinto secundario (cuarto de roedores) durante 24 horas, se registró al inicio de la jornada de trabajo la temperatura máxima y mínima, en grados Celsius. Luego se tomó la diferencia entre la temperatura máxima y mínima, se escogió para el análisis la más significativa dentro de las tres semanas de lactancia. Paralelamente, la variable humedad relativa fue medida con los mismos termohigrómetros durante 24 horas.

\section{Análisis estadístico}

Para el análisis de los datos se utilizaron dos métodos: uno para la comparación de medias de las crías destetadas en los dos grupos en el primer y segundo parto de las hembras; y otro para medir la contribución de diferentes variables comparado al número de cambios de los animales (frecuencia de limpieza), incluyendo temperatura, humedad, número de parto de las hembras, para verificar si alguna de estas variables era significativa en la cantidad de crías destetadas en el grupo control y tratamiento. Este análisis se realizó sólo para las primeras tres semanas de las crías hasta el destete.

El contraste de hipótesis y el cálculo de potencia estadística se analizan con el paquete estadístico JPM. Para analizar los factores de temperatura, humedad y número de partos se opta por construir un modelo de regresión logística.

El modelo de regresión logística que fue desarrollado por estudiantes de la escuela de estadística de la Universidad de Costa Rica:

Ecuación 1. Modelo de regresión logística

$$
\operatorname{In}\left(\frac{p}{1-p}\right)=\beta_{0}+\beta_{1} x_{1}+\beta_{1} x_{2}+\beta_{3} x_{3}+\beta_{4} x_{4}+\varepsilon
$$

Donde

$\mathrm{x}_{1}$ : cambio de cama

$\mathrm{x}_{2}$ : cantidad de parto

$\mathrm{x}_{3}$ : temperatura

$\mathrm{x}_{4}$ : humedad

$\operatorname{In}\left(\frac{p}{1-p}\right)=$ Probabilidad de significancia (variable independiente)

$\mathcal{E}=$ Estimador de la variable

$\mathcal{E}=$ Residuo

$\beta_{0}=$ Variable dependiente

Es importante resaltar que el modelo descrito anteriormente presenta un buen ajuste. Esto se comprobó con la prueba de Pearson de bondad de ajuste (en la que la hipótesis nula para esta prueba es que el modelo está ajustado). Por tanto, no hay suficiente evidencia estadística como para rechazar la hipótesis nula de que el modelo tiene un buen ajuste. Las razones de ventaja y los p-Value de cada una de las variables, calculadas a partir del modelo de regresión anterior, pueden observarse en el modelo calculado.

\section{Resultados}

\section{Primer parto}

En la tabla 1 se presenta el número de crías nacidas y destetadas, el porcentaje de supervivencia del número de crías nacidas y destetadas de las 28 madres del primer parto.

Tabla 1. Parámetros de animales nacidos, destetados, tasa de natalidad y supervivencia para el primer parto

\begin{tabular}{|l|c|c|c|c|}
\hline $\begin{array}{c}\text { Tipo de } \\
\text { animales }\end{array}$ & $\begin{array}{c}\text { Animales } \\
\text { nacidos }\end{array}$ & $\begin{array}{c}\text { Animales } \\
\text { destetados }\end{array}$ & Natalidad & $\begin{array}{c}\text { Supervivencia } \\
(\%)\end{array}$ \\
\hline Tratamiento & 308 & 271 & 11,0 & 88,0 \\
\hline Control & 291 & 255 & 10,4 & 87,6 \\
\hline
\end{tabular}

Fuente: elaboración propia

Durante el primer parto se obtiene para el grupo control al momento del destete una media de 9,1 crías por hembra reproductora, correspondiente a una población de 28 madres, mientras que para el grupo tratamiento se obtuvo una media de 9,7 crías por hembra de una población de 28 madres reproductoras (tablas 2 у 3$)$. 
Tabla 2. Comparación de la población del grupo control con el grupo tratamiento en el primer parto de ratas

\begin{tabular}{|l|c|c|}
\hline \multirow{2}{*}{ Variable estadística } & \multicolumn{2}{|c|}{ Población } \\
\cline { 2 - 3 } & Grupo control & Grupo tratamiento \\
\hline Media & 9,95 & 10,15 \\
\hline Varianza & 28,26 & 36,13 \\
\hline N (población) & 28 & 28 \\
\hline
\end{tabular}

Fuente: elaboración propia

Tabla 3. Prueba de hipótesis de la población del grupo control con el grupo tratamiento en el primer parto

\begin{tabular}{|l|c|c|c|}
\hline \multicolumn{1}{|c|}{$\begin{array}{c}\text { Prueba usando dos } \\
\text { colas }\end{array}$} & Probabilidad & $\mathrm{H}_{0} \Phi$ & $\mathrm{H}_{1} \Psi$ \\
\hline Var. iguales & 0,91 & $\mu_{1}=\mu_{2}$ & $\mu_{1}<>\mu_{2}$ \\
\hline Var. distintas & 0,91 & $\mu_{1}=\mu_{2}$ & $\mu_{1}<>\mu_{2}$ \\
\hline & & & \\
\hline Prueba usando una cola & Probabilidad & & \\
\hline Var. iguales & 0,46 & $\mu_{1}=\mu 2$ & $\mu_{1}<\mu_{2}$ \\
\hline Var. distintas & 0,46 & $\mu_{1}=\mu 2$ & $\mu_{1}<\mu_{2}$ \\
\hline
\end{tabular}

Fuente: elaboración propia

\section{Segundo parto}

En la tabla 4 se presenta el número de crías nacidas y destetadas, y el porcentaje de supervivencia del número de crías nacidas y destetadas de las 8 madres del segundo parto.

Tabla 4. Parámetros de animales nacidos, destetados, tasa de natalidad y supervivencia para el segundo parto

\begin{tabular}{|l|c|c|c|c|}
\hline $\begin{array}{c}\text { Tipo de } \\
\text { animales }\end{array}$ & $\begin{array}{c}\text { Animales } \\
\text { nacidos }\end{array}$ & $\begin{array}{c}\text { Animales } \\
\text { destetados }\end{array}$ & Natalidad & $\begin{array}{c}\text { Supervivencia } \\
(\%)\end{array}$ \\
\hline Tratamiento & 109 & 75 & 13,6 & 68,8 \\
\hline Control & 95 & 78 & 11,9 & 82,1 \\
\hline
\end{tabular}

Fuente: elaboración propia

Durante el destete, se obtiene una media para el grupo control de 9,8 crías por hembra, correspondien- te de una población de 8 madres, mientras que para el grupo tratamiento se obtuvo una media de 9,4 crías por hembra de una población de 8 madres.

Tabla 5. Comparación de la población del grupo control con el grupo tratamiento en el segundo parto

\begin{tabular}{|c|c|c|}
\hline \multirow{2}{*}{ Estadística } & \multicolumn{2}{|c|}{ Población } \\
\hline & Grupo control & Grupo tratamiento \\
\hline Media & 9,75 & 9,38 \\
\hline Varianza & 24,79 & 35,98 \\
\hline n(población) & 8 & 8 \\
\hline
\end{tabular}

Fuente: elaboración propia

Tabla 6. Pruebas de hipótesis de la población del grupo control con el grupo tratamiento en el segundo parto

\begin{tabular}{|l|c|c|c|}
\hline \multicolumn{1}{|c|}{$\begin{array}{c}\text { Prueba usando dos } \\
\text { colas }\end{array}$} & Probabilidad & $\mathbf{H}_{0}{ }^{\Phi}$ & $\mathbf{H}_{1}{ }^{\Psi}$ \\
\hline Var. iguales & 0,893708782 & $\mu_{1}=\mu_{2}$ & $\mu_{1}<>\mu_{2}$ \\
\hline Var. distintas & 0,893773816 & $\mu_{1}=\mu_{2}$ & $\mu_{1}<>\mu_{2}$ \\
\hline & & & \\
\hline Prueba usando una cola & Probabilidad & & \\
\hline Var. iguales & 0,446854391 & $\mu_{1}=\mu_{2}$ & $\mu_{1}<\mu_{2}$ \\
\hline Var. distintas & 0,446886908 & $\mu_{1}=\mu_{2}$ & $\mu_{1}<\mu_{2}$ \\
\hline
\end{tabular}

Fuente: elaboración propia

En el caso del análisis estadístico realizado de regresión logística, se hace un monitoreo de la variable cambio de temperatura durante todo el tiempo de la investigación y se obtiene una variabilidad máxima de 10 ${ }^{\circ} \mathrm{C}$ y una mínima de $6,7^{\circ} \mathrm{C}$.

De la estimación del modelo logístico, el único coeficiente de regresión que resulta significativo (valor $\mathrm{p}$ menor que 0,05 ) es el asociado a la variable cantidad de parto (valor $\mathrm{p}=0,012$ ). Las demás variables no fueron significativas, pues presentaron valores de $\mathrm{p}$ mayores al p de significancia como se muestra en la ecuación 2 .

Ecuación 2. Cálculo del modelo regresión logística (entre paréntesis valores de p).

$$
\operatorname{In}\left(\frac{p}{1-p}\right)=\begin{gathered}
0,9643 \\
(0,617)
\end{gathered}-\begin{gathered}
0,3143 X_{2} \\
(0,568)
\end{gathered}-\begin{gathered}
1,4622 X_{2} \\
(0,012)
\end{gathered}+\begin{gathered}
0,0559 X_{3} \\
(0,852)
\end{gathered}+\begin{gathered}
0,0614 X_{4} \\
(0,293)
\end{gathered}
$$

Donde

$\mathrm{x}_{1}$ : cambio de cama

$\mathrm{x}_{2}$ : cantidad de parto

$\mathrm{x}_{3}$ : temperatura

$\mathrm{x}_{4}$ : humedad 


\section{Discusión}

Este estudio se realiza para generar información que pueda proporcionar una guía de normas basadas en bienestar animal de las ratas Sprague Dawley para producción. De acuerdo con los resultados obtenidos en el estudio y con su evaluación estadística se evidencia que no hay diferencia significativa entre la frecuencia de cambio del recinto primario de las ratas Sprague Dawley de una vez semanal a dos veces semanales, tomando como parámetro el número de crías destetadas. Al comparar las medias de los grupos control y tratamiento no se encontraron diferencias - estadísticamente significativas - en la producción de ratas Sprague Dawley (tabla 2, 3, 5 y 6) del primer y segundo parto, con un régimen de limpieza de una vez semanal o dos limpiezas por semana.

Algunas investigaciones indican que el cambio frecuente de cama en ratas de laboratorio con crías provoca una pérdida de las señales olfativas maternas y por supuesto un aumento del estrés estimulado por la manipulación humana [9]. Esta variable en los primeros días de nacimiento fue minimizada por los técnicos, por el método en que se transportan el nido hecho por las madres, de las cajas sucias a las cajas limpias. Estos son días críticos en el cuido materno, por lo que se mantienen las señales de hormonas intactas y no se expone en gran parte a la pérdida del vínculo materno. Es importante decir que esto sólo se realiza en los primeros días de nacimiento de las crías y de igual forma para el grupo tratamiento y el grupo control; en consecuencia, al aplicarse a los dos grupos es una variable que no agrega una variabilidad mayor al estudio, pues afecta en la misma proporción en ambos grupos. Se debe recordar que el objetivo es evaluar el efecto del cambio del régimen de cambio de las jaulas de los animales.

De la estimación del modelo logístico el coeficiente de regresión que resultó significativo fue el asociado a la variable cantidad de parto $(\mathrm{p}=0,012)$. Por consiguiente, se obtiene que esta variable disminuye la probabilidad de sobrevivencia de las crías de las ratas Sprague Dawley para las condiciones del experimento; en futuros estudios se debería ampliar la muestra para verificar que tan importante es esta influencia en la producción. Por factores biológicos, esta especie de rata, al igual que otras especies animales, se ve influenciada por el historial de fecundidad de la madre: conforme aumenta la cantidad de partos que tiene la madre, la sobrevivencia de las nuevas crías va disminuyendo. La madre primeriza se caracteriza por tener mayor cantidad de crías (experiencias propias de la unidad), y tener más posibilidad de resistir las primeras tres semanas de lactancia que son cruciales en su sobrevivencia; no obstante, algunas madres primerizas en el estudio no presentaron buenas habilidades de cuido materno y algunas tuvieron un número de crías bajo, pero en promedio las madres primerizas tuvieron una supervivencia mayor comparada con las madres en un segundo parto. Por lo mencionado, los resultados obtenidos en este estudio concuerdan con trabajos realizados anteriormente, donde tampoco se logró identificar la relación entre frecuencia de limpieza de cama y sobrevivencia de la camada $[1,14]$.

$\mathrm{Al}$ analizar las temperaturas y las humedades se muestra que las variaciones observadas en este estudio no influyen directamente en el número de crías destetadas. Debido a que las variables temperatura y humedad son importantes para el metabolismo y sobrevivencia de las ratas en condiciones de laboratorio, diversos estudios indican que la temperatura de mantenimiento de las ratas debe estar entre los 19 y $24^{\circ} \mathrm{C}$, es decir, la variación permitida es de 4 a $5{ }^{\circ} \mathrm{C}$. $[4,5]$. Los cambios en la temperatura en nuestro estudio se encuentran entre 6 y 10 ${ }^{\circ} \mathrm{C}$ y los datos correspondientes a la humedad entre $21 \mathrm{y}$ $45 \%$, datos que dificultan llegar a una conclusión en relación con el efecto de la temperatura y la humedad. Sin embargo, dichos cambios, al ser tan fuertes, deberían reflejarse en un aumento de la muerte de las crías, lo cual no sucede. Es importante resaltar que estos resultados se pueden deber a la ausencia de control en la temperatura y humedad durante las noches, pero debido a que los cambios no están alejados inclusive de los datos ideales para temperatura y humedad que se indican en la guía nacional, por lo que, como se muestra en los resultados, no influyen [15].

Con base en los datos obtenidos en la investigación, se determina que en las condiciones de este trabajo no hay diferencias significativas al realizar la limpieza de las cajas una o dos veces a la semana en la cantidad de ratas Sprague Dawley producidas.

\section{Agradecimiento}

Se agradece a los estudiantes de Estadística de la Universidad de Costa Rica por su valioso apoyo en los análisis, y a los técnicos especializados del LEBi por la colaboración en la toma de datos. Al taller de publicaciones científicas Instituto de Investigaciones en Salud de la Universidad de Costa Rica y University of Miami, Estados Unidos (taller de publicaciones INISA-UM), por su asesoría para la elaboración de este artículo. 


\section{Referencias}

[1] Burn CC, Mason GJ. Absorbencies of six different rodent beddings: commercially advertised absorbencies are potentially misleading. Lab Anim. Ene 2005; 39(1): 68-74.

[2] Health and Safety Executive. Control of Sub-stances Hazardous to Health (COSHH) Approved Codes of Practice. London: HMSO; 1994.

[3] Broderson JR, Lindsey JR, Crawford JE. The role of environmental ammonia in respiratory mycoplasmosis of rats. Am J Pathol. 1976; 85(1): 115-130.

[4] Gamble MR, Clough G. Ammonia build-up in animal boxes and its effect on rat tracheal epithelium. Lab Anim. Abr 1976; 10(2): 93-104.

[5] Serrano LJ. Carbon dioxide and ammonia in mouse cages: effects of cage covers, population, and activity. Lab Anim Sci. Feb 1971; 21(1): 75-85.

[6] Van Winkle TJ, Balk MW. Spontaneous corneal opacities in laboratory mice. Lab Anim Sci. Jun 1986; 36(3): 248-55.

[7] Berg RW, Buckingham K, Stewart RL. Etiologic Factors in Diaper Dermatitis: The Role of Urine. Pediatr Dermatol. Feb 1986; 3(2): 102-6.

[8] St Claire MB, St Claire MC, Davis JA, Chang L, Miller GF. Barrier film protects skin of incontinent rats. Contemp Top Lab Anim Sci. Sep 1997; 36(5): 46-8.
[9] Burn C, Peters A, Day MJ, Mason GJ. Long-term effects of cage-cleaning frequency and bedding type on laboratory rat health, welfare, and handleability: a crosslaboratory study. Lab Anim. Oct 2006; 40(4): 353-70.

[10] Chantry D. The effects of handling and cage changing on breeding mice. J Ani Tech Ass. 1964; 15: 78-80.

[11] Reeb-Whitaker CK, Paigen B, Beamer WG, et al. The impact of reduced frequency of cage changes on the health of mice housed in ventilated cages. Lab Anim. Ene 2001; 35(1): 58-73.

[12] Peters AG, Bywater PM, Festing MFW. The effect of daily disturbance on the breeding perfor-mance of mice. Lab Anim. Abr 2002; 36(2): 188-92.

[13] Cisar CF, Jayson G. Effects of frequency of cage cleaning on rat litters prior to weaning. Lab Anim Care. 1967; 17: $215-17$

[14] Burn C, Mason G. Effects of cage-cleaning frequency on laboratory rat reproduction, cannibalism, and welfare. Appl Anim Behav Sci. Nov 2008; 114(1); 235-247.

[15] Ministerio de Ciencia y Tecnología. Guía para el manejo de animales de laboratorio: Comité Técnico Nacional sobre Utilización de Animales de Laboratorio. 1998; La Gaceta. (44). 